\title{
An inexpensive circuiı for monitoring photomultiplier anode current
}

\author{
Dov Frishman and Carl Akerlof \\ Randall Laboratory of Physics, Unicersity of Michigan, Ann Arbor, MI 48109-1120, USA
}

Received 16 August 1991

An 8-channel current-to-frequency converter module has been developed for measuring photomultiplier anode currents in the range of 1-100 $\mu \mathrm{A}$. Although this circuit was designed specifically for monitoring photomultiplier currents in a ground-based very high energy gamma-ray telescope, it would be useful in many other applications where cost is a consideration. An interesting consequence of this technique is that, by measuring the pulse-to-pulse time interval, it is possible to synthesize the kind of measurements usually performed with lock-in amplifiers. Tests show that with a few seconds integration time, a $1 \mathrm{kHz}$ modulated signal can be detected at a level of $10^{-5}$ of the ambient current.

\section{Introduction}

Although photomultipliers have large bandwidth and dynamic range, they are susceptible to damage if the average dc anode current exceeds levels of the order of $100 \mu \mathrm{A}$. Even in applications where these limits are not approached, knowledge of the dc current is useful for understanding the background fluctuation noise spectrum. In the particular case of ground-based very high energy gamma-ray astronomy, there have been numerous reports of short term bursts of air showers which have been attributed to radiation from various stellar objects. It is quite possible, instead, that terrestrial or solar effects induce a general sky brightening that is responsible for a general increase in trigger rate. To disentangle these problems, a simple dc current-to-frequency circuit has been designed for a new Cherenkov air shower telescope, GRANITE, which is being completed at Mt. Hopkins in Arizona [1]. The principal requirements of this circuit were economy and stable linear performance over the range

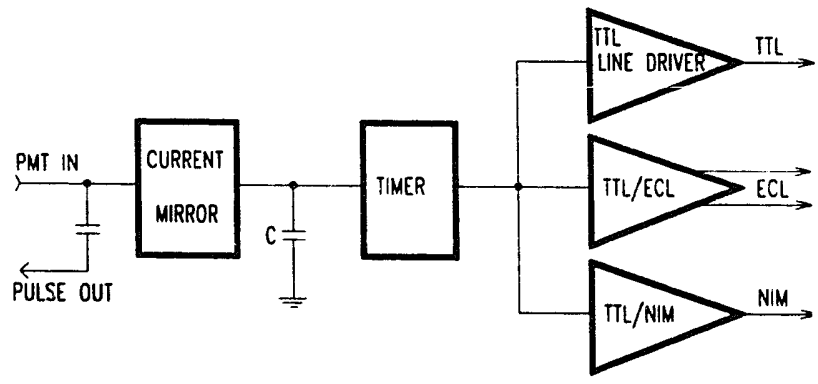

Fig. 1. Block diagram of the photomultiplier anode current monitor. of 1-100 $\mu \mathrm{A}$. The circuit was specifically designed to yield pulse rates from $100 \mathrm{~Hz}$ to $10 \mathrm{kHz}$ which could be accepted by a low-cost 32-channel scaler module engineered for the GRANITE project. A block diagram is shown in fig. 1.

\section{Circuit description}

The circuit design was predicated on operating the photomultiplier with negative high voltage so that the anode runs at close to ground potential. A $10 \mu \mathrm{A}$ current charging a $0.01 \mu \mathrm{F}$ capacitor would generate a voltage of $1000 \mathrm{~V} / \mathrm{s}$, ideal for a timer circuit such as an NE555. However, this cannot be directly implemented since the chip requires a positive charging current for normal operation. This problem was solved by using a current mirror formed by two monolithic PNP transistors to copy the negative current flow from the photomultiplier to a positive current flow into the timing capacitor. For our application, an ICM7555 chip was selected for the timing function. This is a CMOS version of the NE555 manufactured by Harris. The complete circuit is shown in fig. 2.

Fast pulses typically of the order of $5 \mathrm{~ns}$ are shunted by a $0.01 \mu \mathrm{F}$ capacitor to the ac output while the dc current is injected through a $2 \mathrm{k} \Omega$ resistor to the current mirror consisting of two PNP transistors on an MPQ2907 chip. The output of the mirror charges the $0.047 \mu \mathrm{F}$ capacitor connected to the ICM7555. The width of the pulses generated by the ICM7555 is governed by the product of the $200 \Omega$ resistance and the $0.0047 \mu \mathrm{F}$ capacitor. The ICM7555 drives three different outputs. TTL and differential ECL replicas of the 


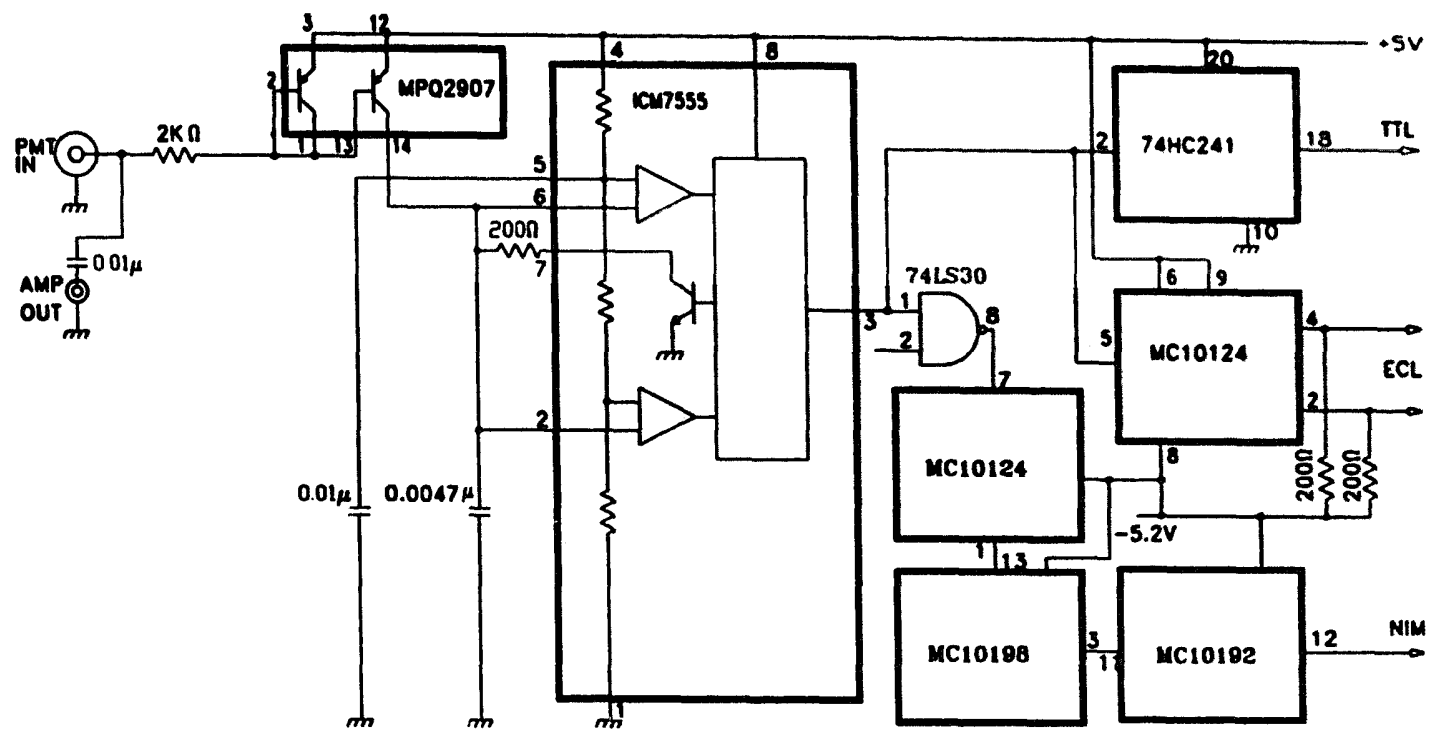

Fig. 2. Schematic diagram of one current monitor channel.

ICM7555 pulse train are generated by $74 \mathrm{HC} 241$ and MC10124 chips. The TTL signals are intended to drive a remote display while the differential ECL pulses are counted by a multichannel scaler module. In addition, pulses from all eight channels are logically summed by a 74LS30, translated to ECL by an MC10124 and shaped to 50 ns width by an MC10198. NIM level output is driven by an MC10192. It is intended that these summed pulse trains can be further combined with signals from similar modules to generate a frequency proportional to the total current in the photomultiplier array. By sensing the total pulse rate, an automatic protection circuit can trip the photomultiplier high voltage supply before tube damage occurs.

The 8-channel multiplicity was chosen to match an amplifier-discriminator module that has been described in a previous article [2]. The printed circuit board dimensions are $233.4 \mathrm{~mm} \times 160.0 \mathrm{~mm}$, corresponding to the VME $6 \mathrm{U}$ size. Four conducting layers were required, two for signal and two for ground and power.

\section{Performance}

The performance of the current mirror chin is the most crucial aspect of the circuit. Since both transistors are on the same substrate, their circuit parameters stay closely matched as the ambient temperature varies. Most of the tests were performed with a HewlettPackard 64111D digital oscilloscope and a HewlettPackard 6114A precision power supply. The accuracy and linearity of the circuit were tested by injection of a known current and measurement of the output frequency. Fig. 3 shows the extreme curves and the aver-

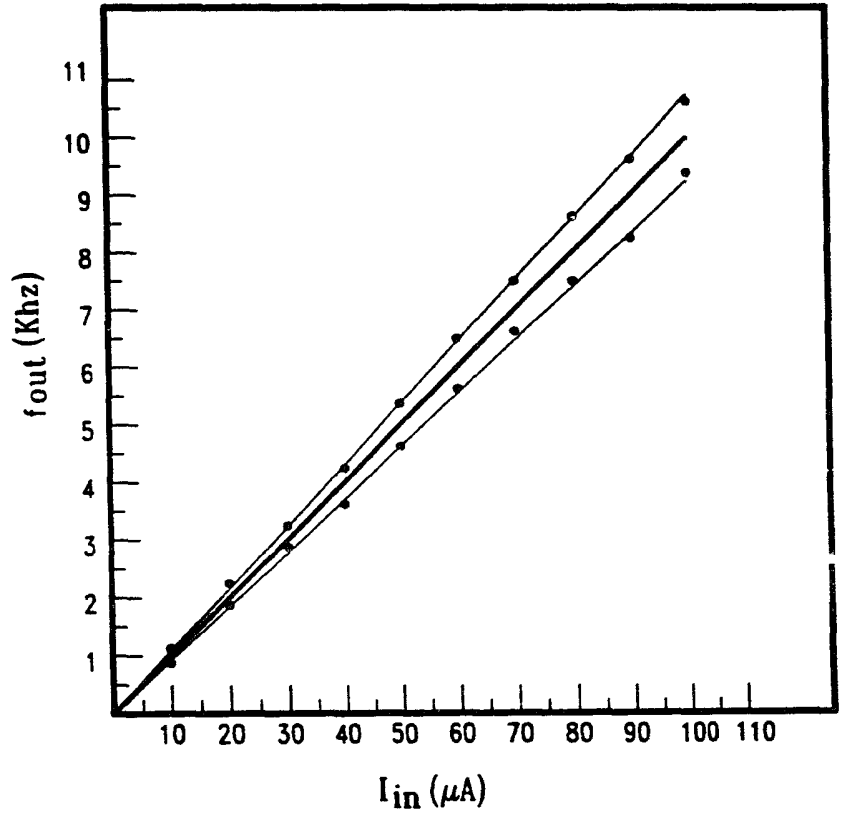

Fig. 3. Calibration curves for 48 current monitor channels. The upper and lower curves represent the extremes of behavior. The central line depicts the system average.

age for 48 channels. The channel-to-channel variation spanned a total range of $\pm 6 \%$ while the linearity was considerably better. No attempt was made to match the slopes by selecting the ICM7555 charging capacitance. The current measurement range is dependen: on the current mirror performance and the ICM7555 leakage current. It is possible with this circuit to operate with anode currents which range from less than 1 $\mu \mathrm{A}$ to greater than $1 \mathrm{~mA}$.

The short-term stability of the ICM7555 pulse train was accurately measured for an injected current of 14 
$\mu \mathrm{A}$. The time sequence of 32768 consecutive pulses was recorded with $50 \mathrm{~ns}$ accuracy by a special CAMAC $20 \mathrm{MHz}$ event clock module designed for precision time series measurements. Over the total sample, the average time between pulses was $0.73 \mathrm{~ms}$ with a standard deviation of less than $0.3 \mu \mathrm{s}$. The data was then analyzed in 3-s segments, each comprising 4092 consecutive time intervals. The short-term behavior was checked by grouping the 4092 intervals into $1023 \mathrm{seg}-$ ments of four measurements each. Two independent sums were computed from this set by adding separately the 1023 first and third elements of each segment. The second and fourth elements were ignored. The two sums always agreed to an accuracy of better than one part in $10^{5}$. This suggests that if the initial current was periodically modulated by a small signal it could be detected at a level comparable with the performance of commercial lock-in amplifiers. The advantage of the method described here is that the analog components are quite cheap and the differencing operation can be performed in the time domain where digital techniques can be carried out easily with a simple microprocessor. Since this technique assumes that the time interval measurements are captured in digital memory, the signal phase and amplitude can be simultaneously recovered.

The 8-channel module requires $100 \mathrm{~mA}$ of $+5 \mathrm{~V}$ power and $400 \mathrm{~mA}$ at $-5 \mathrm{~V}$. The power supply is shared by the amplifier-discriminator modules [2] which accept the fast pulse signals passed by this circuit.

\section{Conclusion}

A photomultiplier current monitor based on current to frequency conversion provides a simple and inexpensive means of recording values in the range of 1 to 100 $\mu \mathrm{A}$. The performance is more than adequate for most applications. The possibility of phase-sensitive detection makes this circuit an interesting candidate for detecting small periodically modulated signals.

\section{Acknowledgements}

The authors thank John Mann for his helpful suggestions concerning this design. This work was supported by the U.S. Department of Energy contract DE-AC02-76ER01112.

\section{References}

[1] C.W. Akerlof et al., Proc. SPIE 33rd Annu. Int. Symp. EUV, X-Ray and Gamma-Ray Instrumentation for Astronomy and Atomic Physics, San Diego, CA, USA, August 7-11, 1989, SPIE Conf. Proc. Vol. 1159, p. 270.

[2] H. Levy, D. Frishman and C. Akerlof, Nucl. Instr. and Meth. A292 (1990) 715. 